\section{Vantagens da técnica de quadrantectomia em cadelas com neoplasias mamárias}

\author{
Advantages of quadrantectomy in bitches with mammary \\ neoplasm
}

Saulo Andrade Caldas'*, Mariana Correia Oliveira², Lucinéia Costa Oliveira ${ }^{3}$, Kelly Regina Freitas Freire ${ }^{4}$, Ana Carolina Campos ${ }^{3}$, Ana Paula de Castro Pires², Vivian de Assunção Nogueira ${ }^{5}$ \& Gabriela de Carvalho Cid²

'Médico veterinário, Dr, Docente. Departamento de Medicina e Cirurgia Veterinária - DMCV, Instituto de Veterinária, Universidade Federal Rural do Rio de Janeiro - UFRRJ, Seropédica, RJ, Brasil

${ }^{2}$ Médicas veterinárias, MSc, Doutorandas. Programa de Pós-graduação em Medicina Veterinária (Patologia e Ciências Clínicas) - PPGMV, Universidade Federal Rural do Rio de Janeiro - UFRRJ, Seropédica, RJ, Brasil

${ }^{3}$ Médicas veterinárias, Mestrandas. Programa de Pós-graduação em Medicina Veterinária (Patologia e Ciências Clínicas) PPGMV, Universidade Federal Rural do Rio de Janeiro - UFRRJ, Seropédica, RJ, Brasil

${ }^{4}$ Médica veterinária, Residente na área de Cirurgia de Pequenos Animais. Universidade Federal Fluminense - UFF, Niteroi, RJ, Brasil ${ }^{5}$ Médica veterinária, Dr, Docente. Departamento de Epidemiologia e Saúde Pública - DESP, Instituto de Veterinária, Universidade Federal Rural do Rio de Janeiro - UFRRJ, Seropédica, RJ, Brasil

\section{Resumo}

Diversos modelos cirúrgicos são propostos para a retirada das neoplasias mamárias em cadelas, no entanto, os estudos de comparação entre as técnicas são escassos. O objetivo principal deste trabalho foi estabelecer um modelo de procedimento clínico e cirúrgico no tratamento das neoplasias mamárias em cadelas, similar ao observado em mulheres e designado como quadrantectomia. Foram atendidas 154 cadelas no Serviço de Obstetrícia do Hospital Veterinário da UFRRJ no período compreendido entre março de 2014 a dezembro de 2015. Destas, 141 cadelas com diversos tipos de neoplasias mamárias foram operadas. Na avaliação pré-operatória foram realizados exames complementares para determinação do estadiamento tumoral (Sistema TNM) e do risco cirúrgico. A pré-anestesia foi realizada com Acepromazina 0,2\% (0,2 mg/kg, IM) ou Diazepan 0,5\% (0,5 mg/kg, IM). A indução anestésica geral foi feita com Diisopropilfenol 1\% (2,0 a 8,0 mg/kg, IV) e a manutenção anestésica geral com Isoflurano ofertado por traqueotubo e diluído em oxigênio a 100\%, em circuito fechado de anestesia. O emprego da técnica de quadrantectomia para exérese de neoplasias mamárias constituiu uma manobra preventiva ao surgimento de novas massas tumorais, comprovada mediante a taxa de sobrevivência de 95,5\%, em comparação com o emprego da técnica de mastectomia unilateral direita ou esquerda ou ainda a mastectomia bilateral total, cuja taxa de sobrevivência foi de 90,5\%. A técnica é facilmente executável, associada ao método de dissecção, uso de dreno cirúrgico e curativo compressivo.

Palavras-chave: cães, mastectomia, tumores, quadrantectomia.

\begin{abstract}
A great number of surgical procedures have been proposed for the treatment of mammary tumors in bitches; however, few surveys have compared the accuracy of different techniques in dogs. Our study aimed to establish a quadrantectomy clinical and surgical protocol for the treatment of mammary tumors in bitches, similar to those performed in women. Between March 2014 and December 2015, 154 bitches were admitted to the Obstetric Sector of the Veterinarian Hospital of the Rural Federal University of Rio de Janeiro. One hundred and forty-one of those bitches presented different variants of mammary tumors and were treated with surgical procedures. Pre-operational evaluation included tumor staging through the TNM system and assessment of surgical risk. The following agents were used throughout the surgical procedures: Acepromazine 0.2\% (0.2 mg/kg, IM) or Diazepam for pre-anesthesia, a Diisopropylphenol $0.5 \%$ (2.0 to $8.0 \mathrm{mg} / \mathrm{kg}$, IV), IM during the general anesthesia induction, and closed-circuit Isoflurane in the stage of management of anesthesia. Quadrantectomy was performed as a maneuver to prevent tumor recurrence. The maneuver proved effective due to the $95.5 \%$ survival rate, compared to the $90.5 \%$ survival rate of right or left unilateral mastectomy and bilateral mastectomy techniques. Quadrantectomy in association with dissection method, surgical drain, and compression bandaging was considered an effective and easily applicable surgical procedure in bitches with mammary tumors.
\end{abstract}

Keywords: dogs, mastectomy, tumors, quadrantectomy.
Como citar: Caldas, S. A., Oliveira, M. C., Oliveira, L. C., Freire, K. R. F., Campos, A. C., Pires, A. P. C., Nogueira, V. A., \& Cid, G. C. (2017). Vantagens da técnica de quadrantectomia em cadelas com neoplasias mamárias. Brazilian Journal of Veterinary Medicine, 39(3), 190-197. doi: 10.29374/2527-2179.bjvm010517

\section{Fonte de financiamento: FAPERJ.}

Conflito de interesses: Os autores declaram não haver conflito de interesses que precisam ser informados.

Recebido: Janeiro 18, 2017

Aceito: Março 16, 2017.

O estudo foi realizado na Universidade Federal Rural do Rio de Janeiro - UFRRJ, Seropédica, RJ, Brasil.

\section{*Correspondência}

Saulo Andrade Caldas

Programa de Pós-graduação em Medicina Veterinária, Instituto de Veterinária,

Universidade Federal Rural do Rio de Janeiro - UFRRJ

Antiga Estrada Rio-São Paulo, BR-465, Km 7 CEP 23891-000 - Seropédica (RJ), Brasil

E-mail:sauloacaldas@hotmail.com 


\section{Introdução}

As neoplasias mamárias são comumente observadas em cadelas não castradas com média de idade de 11 anos (Sleeckx et al., 2011; Toríbio et al., 2012). Diversos fatores estão associados ao aumento do risco de desenvolvimento de tumores mamários nesta espécie, dentre eles, genéticos (Borge et al., 2011), hormonais (Thuroczy et al., 2007) e nutricionais (Lana et al., 2007).

As neoplasias mamárias em cadelas, assim como na mulher, são classificadas em estágios de I a V de acordo com o sistema TNM, que facilita a determinação do prognóstico dos tumores mamários e sua futura escolha de tratamento (Rutteman \& Kirpensteijn, 2003).

Diversas técnicas cirúrgicas têm sido propostas para a remoção de tumores mamários em cadelas, dentre elas, pode-se citar a nodulectomia, a mastectomia simples, a mastectomia regional e as mastectomias uni e bilateral (Lana et al., 2007; Fossum, 2008). O tipo de cirurgia depende da extensão, drenagem linfática, tamanho e localização do nódulo (Sorenmo et al., 2013).

Na mulher existem diversas técnicas utilizadas para a retirada de neoplasias mamárias; a designada quadrantectomia ou mastectomia segmentar é preconizada na remoção de um quadrante ou segmento da glândula mamária onde se localiza um tumor maligno, assim como as margens adjacentes ao tecido normal e os linfonodos regionais (Franco et al., 1997).

O parênquima glandular mamário em cadelas possui uma distribuição vásculo-nervosa diferenciada de origens axilares e inguinais que determina unidade anatômica (Luiz et al., 2002), deste modo, permite denominar de quadrantes mamários ao conjunto de glândulas torácicas e abdomino-inguinais (direita e esquerda), de modo análogo ao aparelho mamário humano (Tiezzi, 2007).

O objetivo principal deste trabalho foi estabelecer um possível modelo de procedimento clínico e cirúrgico no tratamento das neoplasias mamárias em cadelas, similar ao observado nas mulheres e designado como quadrantectomia, assim como avaliar o percentual da taxa de sobrevivência das cadelas submetidas a esta técnica, em um período de acompanhamento clínico de 12 meses.

\section{Materiais e métodos}

No presente estudo foram atendidas 154 cadelas com neoplasias mamárias no Serviço de Obstetrícia do Hospital Veterinário da Universidade Federal Rural do Rio de Janeiro (UFRRJ), no período compreendido entre março de 2014 a dezembro de 2015.

Deste total, 13 cadelas não foram operadas devido ao comprometimento dos linfonodos regionais e/ou metástase à distância.

A realização dos procedimentos adotados foi aprovada pela Comissão de Ética no Uso de Animais - CEUA, protocolo n 040/2014, com consentimento esclarecido de cada participante (tutor do animal) e as normas éticas exigidas foram cumpridas.

Na avaliação pré-operatória foram realizados exames complementares (hemograma completo, bioquímica sérica, eletrocardiograma, ultrasonografia abdominal e exame radiológico do tórax) para determinação do estadiamento tumoral (Sistema TNM) e do risco cirúrgico.

A pré-anestesia foi realizada com Acepromazina 0,2\% (0,2 mg/kg, IM) ou Diazepan 0,5\% (0,5 mg/kg, IM), a indução anestésica geral com Diisopropilfenol 1\% (2,0 a 8,0 mg/kg, IV) e a manutenção anestésica geral com Isoflurano ofertado por traqueotubo e diluído em oxigênio a $100 \%$, em circuito fechado de anestesia.

As técnicas cirúrgicas empregadas consistiram na quadrantectomia torácica direita, esquerda e bilateral e quadrantectomia abdomino-inguinal direita, esquerda e bilateral; e também a mastectomia unilateral direita, esquerda e mastectomia bilateral total (Figura 1).

A escolha da técnica empregada em cada animal foi determinada pela quantidade e localização dos tumores ao longo da cadeia mamária, ou seja, se houvesse tumores somente na região torácica ou abdomino- inguinal, realizava-se a quadrantectomia; se houvesse tumores tanto na região torácica quanto na abdomino- inguinal, a técnica escolhida seria a mastectomia unilateral.

A técnica cirúrgica consistiu na incisão elíptica da pele ao redor do parênquima mamário, com uma margem de 2 a $3 \mathrm{~cm}$ de distância das tetas, o que permitiu a execução de dissecção romba digital com gaze e exérese tumoral e do linfonodo satélite com o mínimo de hemorragia.

A hemostasia das artérias epigástricas superficiais foram realizadas com fio categute cromado 2-0 (Categute, Technofio) ou poliglactina 9102-0 (Vicryl, Shalon). quadrantectomias abdomino-inguinais 

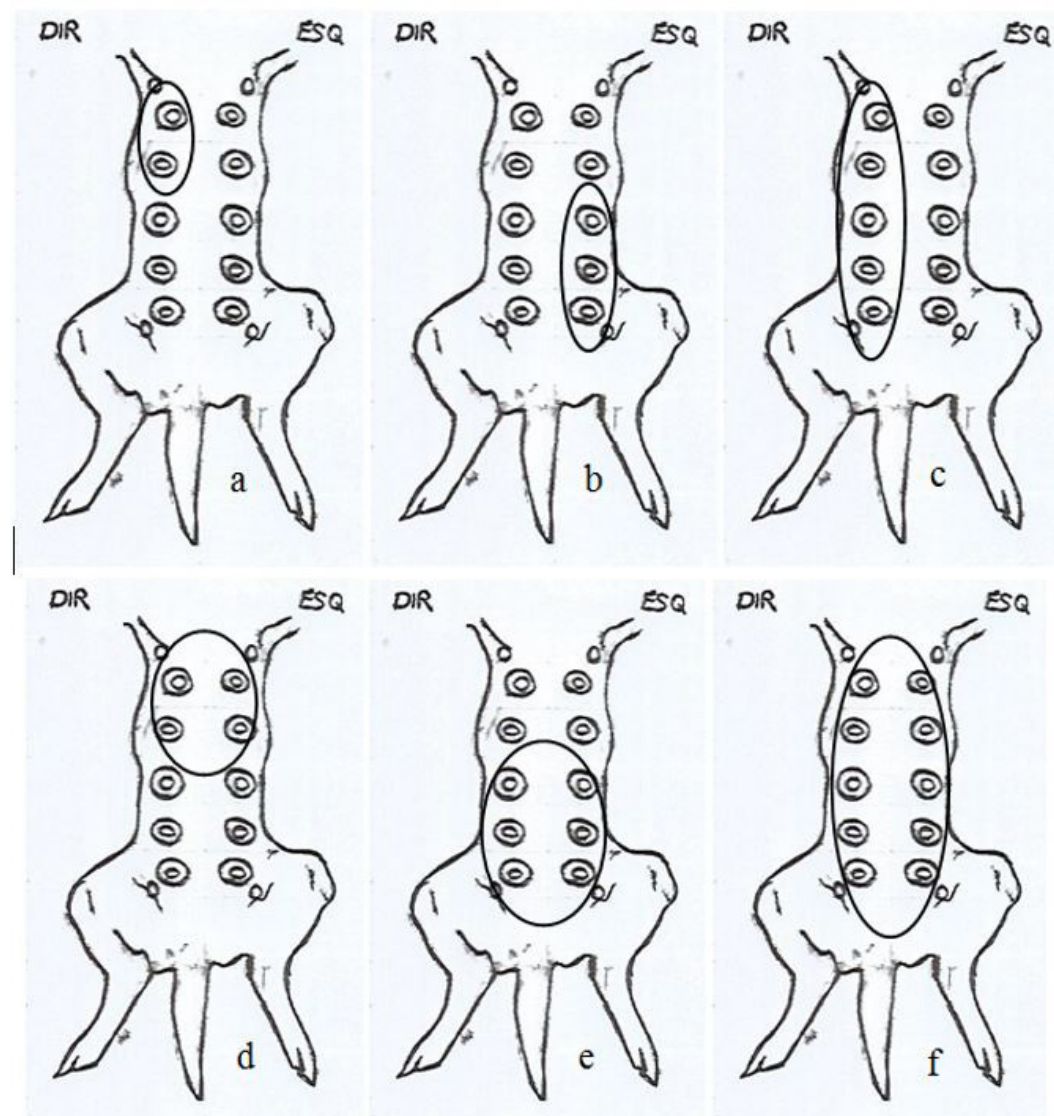

Figura 1. Técnicas de mastectomia empregadas: (a) Mastectomia regional torácica (quadrantectomia); (b) Mastectomia regional abdomino-inguinal (quadrantectomia); (c) Mastectomia unilateral; (d) Mastectomia torácica bilateral (quadrantectomia cranial total); (e) Mastectomia abdomino-inguinal bilateral (quadrantectomia caudal total); e (f) Mastectomia bilateral.

foram introduzidos drenos cirúrgicos de penrose latero-distal à ferida cirúrgica, e posteriormente foi realizada a rafia da pele com fio de nylon monofilamento 2-0 (Nylon, Shalon) sem aplicação de pontos de sutura no plano subcutâneo.

No caso das mastectomias unilaterais introduziu-se um dreno cirúrgico na região abdomino-inguinal, latero-distal à ferida cirúrgica, e posteriormente realizou-se a rafia da pele com fio de nylon monofilamento 2-0, sem aplicação de pontos de sutura no plano subcutâneo.

No pós-operatório, as feridas cirúrgicas foram tratadas diariamente com pomada de sulfadiazina de prata ou merthiolate spray e curativo compressivo realizado com gaze, esparadrapo e atadura de crepon, durante 10 dias. No terceiro dia de pós-operatório foram retirados os drenos das feridas cirúrgicas, e a partir do décimo dia foram retirados os pontos da pele. Os animais operados foram medicados com Amoxicilina e Clavulanato ( $20 \mathrm{mg} / \mathrm{kg}$, VO), de $12 \mathrm{em} 12 \mathrm{~h}$, durante 10 dias; Meloxican (0,1 mg/kg, VO), a cada 24 horas, durante três dias, Tramadol 50 mg ( 1 a 4 mg $/ \mathrm{kg}, \mathrm{VO}$ ), por três a cinco dias, e Dipirona sódica gotas (1 gota/kg, VO), por cinco dias.

\section{Resultados}

A maioria das 141 cadelas operadas apresentava idade entre 8 a 14 anos (82,94\%), média de 11 anos e variação de 4 a 17 anos de idade. As glândulas mamárias mais acometidas por tumores foram as abdominais caudais e inguinais 79/141(56,02\%).

No total, 67/141(47,53\%) cadelas foram submetidas à cirurgia de quadrantectomia. Em 14 destas realizou-se a quadrantectomia torácica esquerda (Figura2), direita e bilateral; e, em 51, a quadrantectomia 
abdomino-inguinal esquerda, direita e bilateral; e em 2 foram realizadas a quadrantectomia torácica direita e abdomino-inguinal esquerda (Figura 3), conforme detalhado na Tabela 1.

Das 67/141(47,6\%) cadelas submetidas à técnica de quadrantectomia, 30/141(21,3\%) e 37/141(26,3\%) apresentaram nódulo único ou múltiplo (Figura 4), respectivamente. Foram observados 518 nódulos nas 141 cadelas operadas.

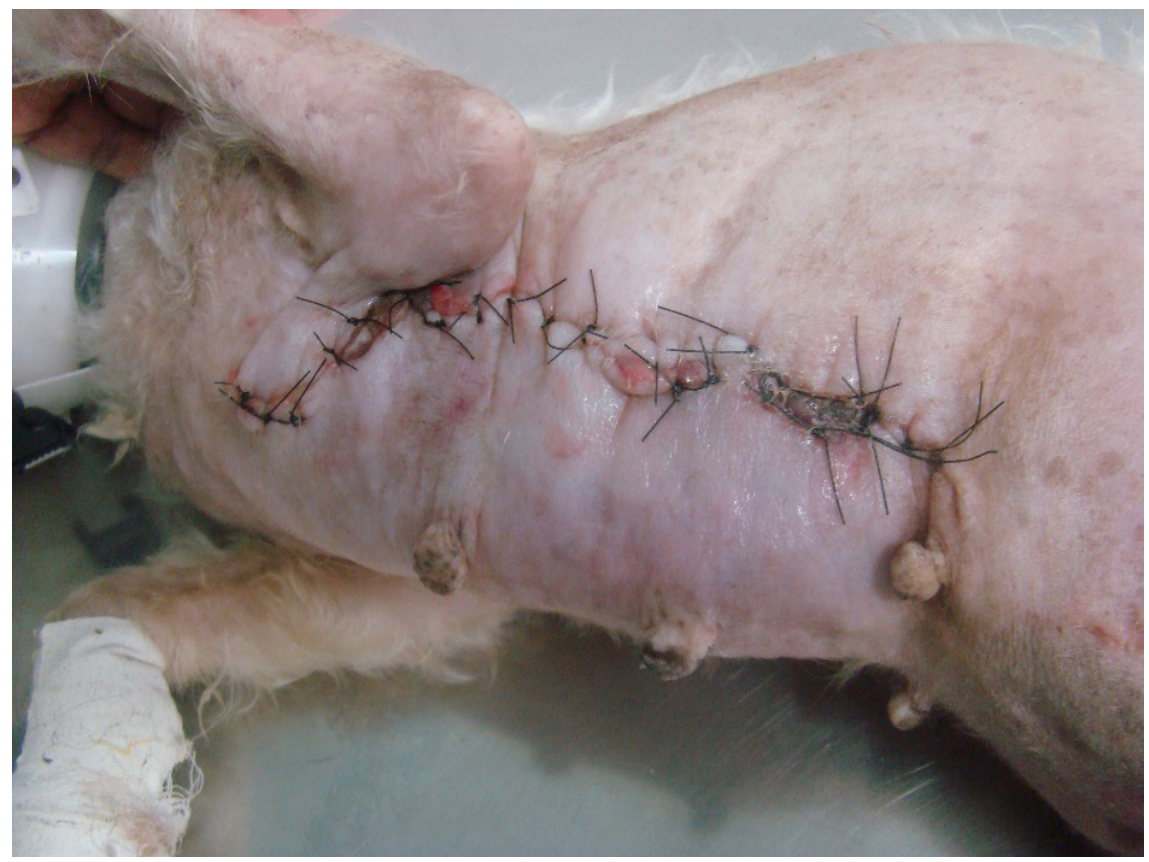

Figura 2. Cadela após retirada de nódulo mamário através da técnica quadrantectomia torácica esquerda.

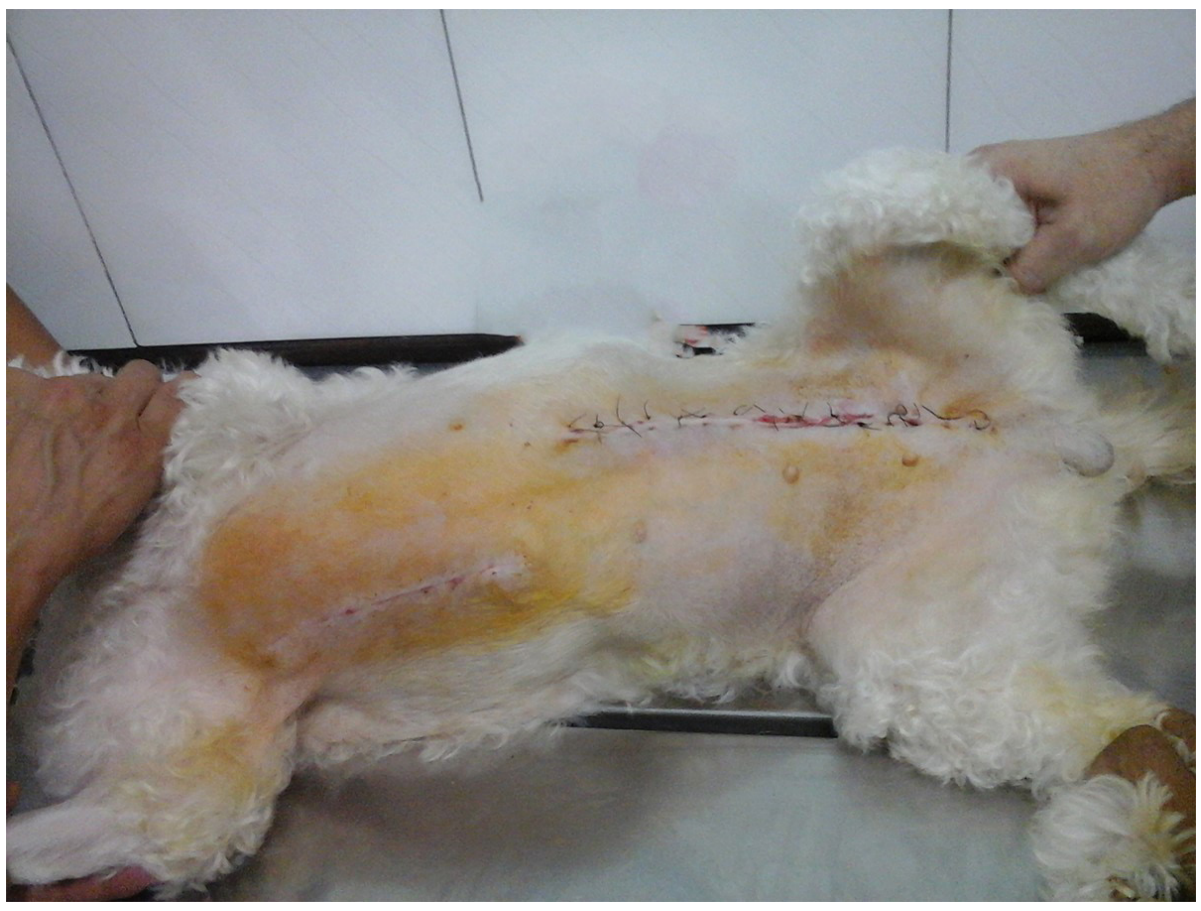

Figura 3. Cadela no pós-cirúrgico da técnica de quadrantectomia torácica direita e quadrantectomia abdomino-inguinal esquerda. 
Tabela 1. Tipos de mastectomias, frequência e porcentual de cirurgias mamárias realizadas em cadelas no Serviço de Obstetrícia do Hospital Veterinário da UFRRJ, entre março de 2014 a dezembro de 2015.

\begin{tabular}{ccc}
\hline Tipos de Mastectomias & $\begin{array}{c}\text { No de } \\
\text { Cadelas }\end{array}$ & Porcentual \\
\hline Quadrantectomia Torácica direita & 4 & 2,8 \\
Quadrantectomia Torácica esquerda & 8 & 5,7 \\
Quadrantectomia Torácica bilateral & 2 & 1,42 \\
Quadrantectomia Abdomino-inguinal direita & 23 & 16,32 \\
Quadrantectomia Abdomino-inguinal esquerda & 16 & 11,35 \\
Quadrantectomia Abdomino- inguinal bilateral & 12 & 8,51 \\
Quadrantectomia torácica direita e Abdomino-inguinal esquerda & 2 & 1,42 \\
Mastectomia Unilateral direita & 38 & 26,95 \\
Mastectomia Unilateral esquerda & 34 & 24,11 \\
Mastectomia Bilateral Total & 2 & 1,42 \\
Total & 141 & 100 \\
\hline
\end{tabular}

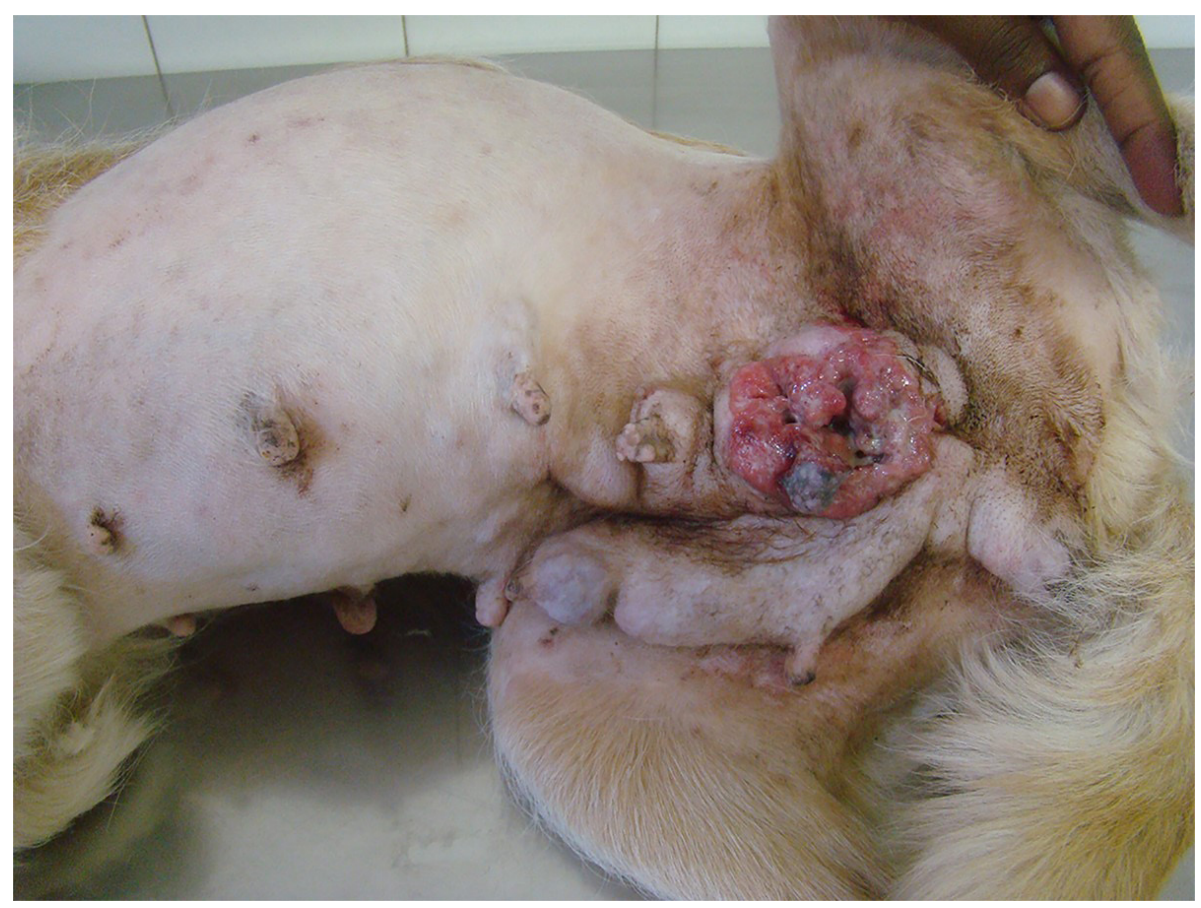

Figura 4. Cadela com nódulos múltiplos e ulcerados em mamas abdomino-inguinais.

Apenas as cadelas com o sistema TNM (T1NOMO, T2NOMO e T3NOMO) foram submetidas à cirurgia (141/154). As demais cadelas foram encaminhadas para o setor de oncologia para a realização de quimioterapia, devido à metástase em linfonodo regional e/ou à distância (Tabela 2).

Das 67/141 cadelas operadas com a técnica de quadrantectomia, 3/67(4,5\%) morreram no período de acompanhamento clínico de 12 meses, devido à ocorrência de metástase pulmonar e esplênica. As demais cadelas não apresentaram recidiva e/ou metástase, durante este tempo de observação, o que resultou em uma taxa de sobrevivência de 95,5\%.

Das 74/141(52,5\%) cadelas operadas com a técnica de mastectomia unilateral direita, esquerda ou bilateral total, 7/74(9,5\%) morreram em um período de observação de 12 meses, devido à 
Tabela 2. Sistema TNM empregado como critério pré-operatório em cadelas com neoplasias mamárias realizadas pelo Serviço de Obstetrícia do Hospital Veterinário da UFRRJ, entre março de 2014 a dezembro de 2015.

\begin{tabular}{cccc}
\hline Sistema TNM & No de cadelas & Estádio & $\begin{array}{c}\text { Porcentual } \\
\text { simples }\end{array}$ \\
\hline T1NOMO & 33 & I & 23,40 \\
T1NOM1 & 1 & V & - \\
T1N1MO & 1 & IV & - \\
T2NOMO & 25 & II & 17,73 \\
T2N1MO & 1 & IV & - \\
T3NOMO & 83 & III & 58,87 \\
T3N1MO & 7 & IV & - \\
T3N1M1 & 2 & V & - \\
T3NOM1 & 1 & V & - \\
Total operadas & 141 & - & 91,5 \\
Total não operadas & 13 & - & 8,5 \\
Total de animais operados e não operados & 154 & - & 100 \\
\hline
\end{tabular}

ocorrência de metástase pulmonar, hepática ou renal ou por incoagulabilidade sanguínea em um caso. As demais cadelas 67/141 não apresentaram recidiva e/ou metástase, durante este período, o que resultou em uma taxa de sobrevivência de 90,5\%.

As principais complicações pós-operatórias evidenciadas nas cadelas foram: seroma em1/141(0,7\%) na quadrantectomia abdomino-inguinal e 6/141(4,25\%) na técnica de mastectomia unilateral. Outras complicações foram a deiscência de sutura em 1/141(0,7\%) na quadrantectomia e 2/141(1,41\%) na mastectomia unilateral; e por último a hemorragia pós-cirúrgica que foi evidenciada em 1/141(0,7\%) das cadelas em que foi utilizada a técnica de mastectomia unilateral.

Quanto aos resultados dos exames histopatológicos, 25/67(37,3\%) e 42/67(62,7\%) cadelas operadas apresentaram neoplasias benignas e malignas, respectivamente. As demais 74 cadelas submetidas às técnicas de mastectomia unilateral e bilateral apresentaram em 26/74(35,1\%) dos casos diagnóstico benigno e 48/74(64,9\%) diagnóstico maligno (Caldas et al., 2016).

Ao comparar as proporções de diagnósticos histopatológicos benignos e malignos, entre a técnica de quadrantectomia e a de mastectomia uni e bilateral, observaram-se semelhanças quanto às porcentagens, 37,3\%; 35,1\% e 62,7\%; 64,9\%, respectivamente.

\section{Discussão}

A idade das cadelas acometidas por neoplasias mamárias variou de oito a quatorze anos, com média de 11 anos, semelhante ao descrito na literatura (Oliveira et al., 2003; Toríbio et al., 2012; Ribas et al., 2012).

Do total de 518 nódulos observados nas 141 cadelas, 396/518(76,4\%) estavam localizados nas mamas abdominal e inguinal, semelhante ao observado em um estudo com 336 fêmeas portadoras de tumores mamários, nas quais as mamas abdominal e inguinal foram as mais acometidas (76,25\%) (Toríbio et al., 2012). Neste contexto, Misdorp et al. (2002), justifica a maior frequência de nódulos nas mamas caudais, devido a maior quantidade de parênquima mamário, o que predispõe a maior alteração proliferativa em resposta hormonal. As 10 cadelas que morreram, apresentaram T3NOMO e o exame histopatológico identificou neoplasias malignas. A categoria T3 do sistema TNM reporta dizer que o nódulo apresenta mais de $15 \mathrm{~cm}$ de diâmetro. Para Misdorp et al. (1999), tumores maiores de 14 cm são mais comumente diagnosticados como malignos. Esta afirmação também se assemelha ao estudo realizado por Yamagami et al. (1996), no qual as cadelas com tumores maiores apresentaram menor taxa de sobrevivência. 
Daleck et al. (1998), evidenciaram uma taxa de sobrevivência em 18/23 cadelas durante um período de acompanhamento de 12 meses. Esses autores utilizaram diferentes técnicas cirúrgicas, inclusive a de mastectomia regional, aqui denominado de quadrantectomia. Porém o estudo não correlacionou a eficiência das diferentes técnicas com a taxa de sobrevivência. No presente estudo, a taxa de sobrevivência foi de 95,5\% nas cadelas submetidas à quadrantectomia e de 90,5\% em cadelas que realizaram a mastectomia unilateral direita, esquerda ou bilateral.

A quadrantectomia baseia-se no conceito de que as glândulas que compartilham a drenagem venosa e linfática podem ser removidas como uma unidade junto com os linfonodos regionais ipsilaterais (Papazoglou et al., 2014), porém a técnica de quadrantectomia aqui empregada diferiu das técnicas preconizadas e já relatadas (Hedlund, 2008; Sleeckx et al., 2011), uma vez que se mostrou de rápida e de fácil execução, com um tempo cirúrgico estimado entre 15 a 75 minutos.

Além do exposto, apenas três cadelas operadas pela técnica de quadrantectomia morreram, em comparação as sete que morreram pela técnica de mastectomia uni e bilateral, em um período de 12 meses. Cabe ressaltar que as proporções de diagnósticos histopatológicos benignos e malignos se assemelham nas cadelas submetidas às duas técnicas.

O emprego de dreno cirúrgico nas quadrantectomias abdomino-inguinal associado ao curativo local compressivo preveniu a formação de seromas e permitiu uma evolução cicatricial favorável. Além disso, soma-se o fato de apresentar um menor custo com anestésico e um menor tempo de exposição aos seus efeitos colaterais.

As principais complicações pós-operatórias foram evidenciadas nas cadelas que realizaram a mastectomia unilateral, das quais incluem o seroma, deiscência de sutura e hemorragia. Estudos sobre neoplasias mamárias em cadelas são frequentes (Itoh et al., 2005; Sleeckx et al., 2011; Caldas et al., 2016) e a extirpação do tumor com margem cirúrgica ainda é a melhor maneira de alcançar a cura (Sleeckx et al., 2011; Sorenmo et al., 2013), porém faltam dados que comparem as técnicas cirúrgicas mais radicais com as conservadoras, as quais inclui a quadrantectomia. Em mulheres esta técnica é bem consolidada e resulta na cura de pacientes mesmo com tumores malignos (Pasta et al., 2015).

\section{Conclusões}

O presente estudo evidenciou a eficácia do tratamento cirúrgico das neoplasias mamárias em cadelas com o emprego da técnica de quadrantectomia e exérese dos linfonodos regionais associado ao método de dissecção, uso de dreno cirúrgico e curativo compressivo. Deste modo, o emprego da técnica de quadrantectomia para exérese de neoplasias mamárias em cadelas constitui uma manobra preventiva ao surgimento de novas massas tumorais.

\section{Referências}

Borge, K. S., Borresen-dale, A. L., \& Lingaas, F. (2011). Identification of genetic variation in 11 candidate genes of canine mammary tumour. Veterinary and Comparative Oncology, 9(4), 241-250. http://dx.doi.org/10.1111/j.14765829.2010.00250.x. PMid:22077404.

Caldas, S. A., Miranda, I. C., Brito, M. F., Nogueira, V. A., Cid, G. C., Costa, S. Z. R., França, T. N., \& Pinto, L. F. (2016). Aspectos clínico-patológicos das neoplasias mamárias em cadelas (Canis familiaris). Revista Brasileira de Medicina Veterinária, 38(Supl. 2), 81-85.

Daleck, C. R., Franceschini, P. H., Alessi, A. C., Santana, Á. E., \& Martins, M. I. M. (1998). Aspectos clínico e cirúrgicos do tumor mamário canino. Ciência Rural,28(1), 95-100. http://dx.doi.org/10.1590/s0103-84781998000100016.

Fossum, T. W. (2008). Cirurgia de pequenos animais (3. ed., p. 1314). São Paulo: Elsevier.

Franco, J. M., Santos, R. A., Castro, K. C., Malfacini, S. S., \& Santoro, C. R. F. (1997). Tratamento cirúrgico do câncer de mama. In J. M. Franco (Ed.), Mastologia: formação de especialista (pp. 203-217). São Paulo: Atheneu.

Hedlund, C. S. (2008). Cirurgia dos sistemas reprodutivo e genital. In: T. W. Fossum (Ed.), Cirurgia de pequenos animais (3. ed., pp. 702-774). Rio de Janeiro: Mosby.

Itoh, T., Uchida, K., Ishikawa, K., Kushima, K., Kushima, E., Tamada, H., Moritake, T., Nakao, H., \& Shii, H. (2005) Clinicopathological survey of 101 canine mammary gland tumors: difference between small-breed dogs and others. The Journal of Veterinary Medical Science, 67(3), 345-347. http://dx.doi.org/10.1292/jvms.67.345. PMid:15805744.

Lana, S. E., Rutteman, G. R., \& Withrow, S. J. (2007). Tumors of the mammary gland. In S. J. Withrow \& D. M. Vail (Eds.), Withrow \& MacEwen's small animal clinical oncology (4. ed., pp. 619-636). St. Louis: Saunders Elsevier. http://dx.doi.org/10.1016/B978-072160558-6.50029-0. 
Luiz, C. R., Miglino, M. A., \& Santos, T. C. (2002). Segmentos anátomo-cirúrgicos arteriais da glândula mamária em cães (Canisfamiliaris, Linnaeus, 1758). Archives of Veterinary Science, 7(1), 27-36. http://dx.doi.org/10.5380/ avs.v7i1.3965.

Misdorp, W., Else, R. W., \& Hellmén, E. (2002). Definitions and explanatory notes. In W. Misdorp (Ed.), WHO histological classification of mammary tumors of the dog and cat (pp. 18-27). Washington: Armed Forces Institute of Pathology.

Misdorp, W., Else, R. W., Hellmén, E., \& Lipscomb, T. P. (1999). Histological classification of mammary tumors of the dog and the cat. In World Health Organization (Ed.), International histological classification of tumors of domestic animals (2. ed., Vol. 7). Washington: Armed Forces Institute of Pathology, American Registry of Pathology, World Health Organization Collaborating Center for Worldwide Reference on Comparative Oncology.

Oliveira, L. O., Oliveira, R. T., Loretti, A. P., Rodrigues, R., \& Driemeier, D. (2003). Aspectos epidemiológicos da neoplasia mamária canina. Acta Scientiae Veterinariae, 31(2), 105-110.

Papazoglou, L. G., Basdani, E., Rabidi, S., Patsikas, M. N., \& Karayiannopoulou, M. (2014). Current surgical options for mammary tumor removal in dogs. Journal of Veterinary Science and Medicine, 2(1), 6.

Pasta, V., Sottile, D., Urciuoli, P., Del Vecchio, L., Custureri, F., \& D'Orazi, V. (2015). Rare chondrosarcoma of the breast treated with quadrantectomy instead of mastectomy: a case report. Oncology Letters, 9(3), 1116-1120. http://dx.doi.org/10.3892/ol.2014.2803. PMid:25663866.

Ribas, C. R., Dornbusch, P. T., Faria, M. R., Wouk, A. F. P. F., \& Cirio, S. M. (2012). Alterações clínicas, epidemiológicas, citológicas, histológicas e estadiamento de cães com neoplasias mamárias. Archives of Veterinary Science, 17(1), 60-68. http://dx.doi.org/10.5380/avs.v17i1.24107.

Rutteman, G. R., \& Kirpensteijn, J. (2003). Tumours of the mammary glands. In J. M. Dobson, B. Lascelles \& X Duncan (Eds.), BSAVA manual of canine and feline oncology (2. ed., pp. 234-242). Gloucester: British Small Animal Veterinary Association.

Sleeckx, N., Rooster, H., Veldhuis Kroeze, E. J. B., Van Ginneken, C., \& Van Brantegem, L. (2011). Canine mammary tumors: an overview. Reproduction in Domestic Animals, 46(6), 1112-1131. http://dx.doi.org/10.1111/j.14390531.2011.01816.x. PMid:21645126.

Sorenmo, K. U., Worley, D. R., \& Goldschmidt, M. H. (2013). Tumors of the mammary gland. In S. J. Withrow, D. M. Vail \& R. L. Page (Eds.), Small animal clinical oncology (5. ed.). St Louis: Elsevier. http://dx.doi.org/10.1016/ B978-1-4377-2362-5.00027-X.

Thuroczy, J., Reisvaag, G. J., Perge, E., Tibold, A., Szilagyi, J., \& Balogh, L. (2007). Immunohistochemical detection of progesterone and cellular proliferation in canine mammary tumors. Journal of Comparative Pathology, 137(2-3), 122-129. http://dx.doi.org/10.1016/j.jcpa.2007.05.005. PMid:17645888.

Tiezzi, D. G. (2007). Cirurgia conservadora no câncer de mama. Revista Brasileira de Ginecologia e Obstetrícia, 29(8), 428-434. http://dx.doi.org/10.1590/S0100-72032007000800008.

Toríbio, J. M. M. L., Lima, A. E., Martins Filho, E. F., Ribeiro, L. G. R., D’Assis, M. J. M. H., Teixeira, R. G., Damasceno, K. A., Cassali, G. D., \& Costa Neto, J. M. (2012). Caracterização clínica, diagnóstico histopatológico e distribuição geográfica das neoplasias mamárias em cadelas de Salvador, Bahia. Revista Ceres, 59(4), 427-433. http://dx.doi. org/10.1590/s0034-737X2012000400001.

Yamagami, T., Kobayashi, T., Takahashi, K., \& Sugiyama, M. (1996). Influence of ovariectomy at the time of mastectomy on the prognosis for canine malignant mammary tumors. The Journal of Small Animal Practice, 37(10), 462-464. http://dx.doi.org/10.1111/j.1748-5827.1996.tb01740.x. PMid:8912239. 